\title{
Precise Prediction of Hurricane Power vs Ocean Temperature
}

\author{
Edward Wolf \\ Department of Applied Physics, Tandon School of Engineering, New York University, Brooklyn, USA
}

\section{Email address:}

ed.wolf@nyu.edu

\section{To cite this article:}

Edward Wolf. Precise Prediction of Hurricane Power vs Ocean Temperature. International Journal of Atmospheric and Oceanic Sciences. Vol. 5, No. 1, 2021, pp. 1-5. doi: 10.11648/j.ijaos.20210501.11

Received: December 7, 2020; Accepted: January 9, 2021; Published: February 23, 2021

\begin{abstract}
It has long been known that hurricanes, the strongest and most destructive atmospheric events, do not occur below a sea surface temperature near $26 \mathrm{C}$. The detailed dependence of hurricane power on ocean temperature is of increasing interest and concern in the prospect of continuing global warming. The hurricane power was usefully quantified by Emanuel in 2005 with the definition and tabulations of the power dissipation index, PDI. This is the integral over the relevant sea areas of the cube of the maximum windspeed, representiting the power dissipated over one year. In his important 2005 paper Emanuel found that the PDI for the North Atlantic increased strongly in recent decades and showed in plots a close correlation of PDI with sea surface temperature. A critical temperature Tc, and a linear T-Tc power law dependence, typical of a continuous phase transition, for hurricanes have prcviously (Wolf, 2020) been inferred from plots of the power dissipation index PDI vs sea surface temperature $\mathrm{T}$. This implies that tropical cyclone formation can usefully be regarded as a second order phase transition of the warm ocean-atmosphere system, driven by disequilibrium in atmospheric water content. We here show that the theory of phase transitions allows a precise prediction of the temperature dependence of hurricane power and windspeed on ocean surface temperature. We find that the wind velocity transition of the hurricane is in the same universality class as the Ising Model, the uniaxial antiferromagenet and the vapor- liquid transition of simple fluids, and shares their critical exponent. An implication for the applicability of potential intensity theory is noted.
\end{abstract}

Keywords: Tropical Cyclone, Power, PDI, Critical Temperature, Universality Class, ISING Model, Continuous Phase Transition

\section{Introduction: The Power Dissipation Index}

An important aspect of the tropical climate is the hurricane. These storms, that generate copious rainfall, occur primarily in the low latitudes of the North Atlantic in the warmest part of the year. The ocean temperature is a key parameter governing these storms as first shown by Palmen [1], 1948, who identified a minimum or threshold temperature, near 26.5 C for such storms. The prediction of the storm strength, closely related to the rainfall, with warming climate, is of great concern and is our topic. We have discovered [2] and further report here that the hurricane results from a phase transition that is essentially similar to that for the vaporliquid transition in simple fluids and to the magnetic phase transition in uniaxial antiferromagnets, all being described as examples of the Ising Model. By discovering this connection we are able to make more accurate predictions of the temperature dependence of hurricane power vs ocean temperature in the Caribbean and other regions supporting tropical cyclones.

This paper concerns the mechanical wind power released by hurricanes and how that power depends on the temperature of the ocean surface. A useful measure of the energy expended by hurricanes is the Power Dissipation Index, PDI, first defined and tabulated by Emanuel [3] 2005:

$$
\mathrm{PDI}=\iint 2 \pi \rho \mathrm{v}^{3} \mathrm{rdrdt}
$$

where $\rho$ is the air density, and the double integral covers all storms in a given ocean basin in one year. Here $\mathrm{v}$ is the peak azimuthal wind speed in the storm in $\mathrm{m} / \mathrm{s}$ and the underlying formula for the power density of the wind at speed $v$ is 


$$
\mathrm{P}=1 / 2 \rho \mathrm{v}^{3} \mathrm{~W} / \mathrm{m}^{2}
$$

It has recently been established (Wolf, 2020) that longstanding extensive and accurate data $[3,4]$ on the Power Dissipation Index PDI reveal a characteristic power law,

$$
\mathrm{PDI}=\mathrm{a}\left(\mathrm{T}-\mathrm{T}_{\mathrm{c}}\right)^{3 \beta}
$$

with PDI rising sharply above a critical value, $\mathrm{T}_{\mathrm{c}}$, of the local ocean temperature, T. A portion of these data are shown [2] in Figure 1. Here $3 \beta=1.0$, appearing as critical exponent and a is arbitrary, a fitting constant.

(From Figure 1, representing data directly replotted from Emanuel [4] 2007, it can be seen that the fit value of a for hurricanes in the North Atlantic over a period of decades is $\mathrm{a}=6.25 \times 10^{11} \mathrm{~m}^{3} / \mathrm{s}^{2} \mathrm{~K}$.)

In more detail, it is seen in Figure 1 that the PDI vs $\mathrm{T}$ data are approximately linear but that the precise exponent, $3 \beta$, could vary perhaps by $10 \%$ from unity, because of the scatter in the difficult measurements. Additionally, Eq. 3 with nominal exponent 1.0 was used to fit all of the data compiled by Emanuel $[3,4]$. Those data cover hurricanes over 70 years in tropical portions of the North Atlantic, North Pacific, West Pacific, and Indian Oceans. Thus, support for the phase transition law Eq. 3 is stronger than that provided alone by the small portion explicitly replotted in Figure 1.

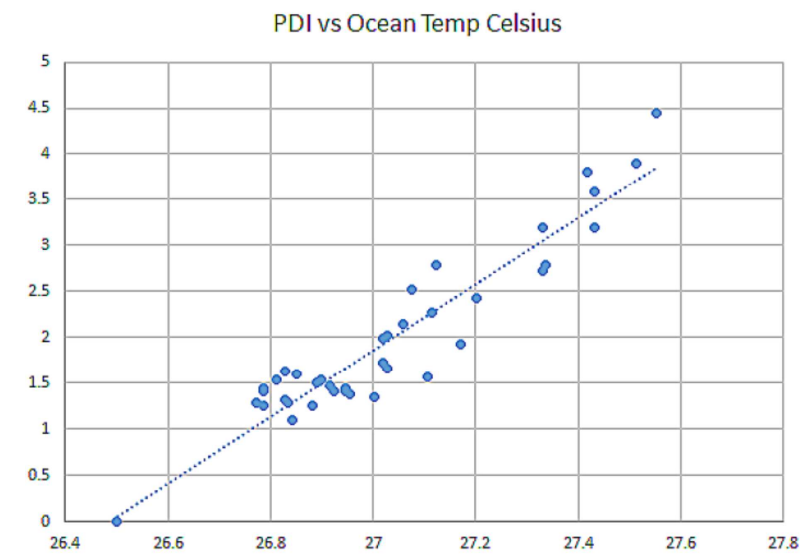

Figure 1. PDI in units $10^{11} \mathrm{~m}^{3} \mathrm{~s}^{-2}$ vs Ocean surface temperature in Celsius. These are direct data points replotted from Emanuel 2007 Figure. 1a, representing the North Atlantic from 1970 to about 2005. The extrapolated line matches the 26.5 C found by Emanuel 2007 Figure 1a and also matches the threshold temperature found by Dare and McBride, 2011 This type of fit appears to be consistent with a phase transition of second order as stated in the text. In this plot, the scatter in the circled data points is an estimate of their uncertainty so error bars are not shown. This figure is reproduced from Wolf 2020.

Measurements of cyclone properties are difficult. A major effort was reported by Emanuel (2005) [3] to define and compile historical values of the Power Dissipation Index PDI. The Power Dissipation Index, is the integral over the duration and area of storms of the cube $\mathrm{V}^{3}$ of the maximum speed $\mathrm{V}$. In this formula, as in the formula for the power of a wind turbine, the cube power comes from the fact that while the kinetic energy per unit mass scales as $\mathrm{V}^{2}$, the relevant amount of energy per second (power) passing through a unit area brings in the additional power of V. The PDI estimates the energy expended and is of interest as a measure of potential damage.

By convention, the PDI integrates all storms in a given ocean basin, such as the North Atlantic, in an appropriate latitude range near but not including the Equator, over one year's time. The quoted values are $\mathrm{m}^{3} / \mathrm{s}^{2}$, but since the time period is one year, the inherent units of the PDI are $\mathrm{m}^{3} / \mathrm{s}^{3}$, corresponding to $\mathrm{V}^{3}$.

In the major works of Emanuel [3, 4], carefully compiled values of PDI, and the sea surface temperature (with an important and carefully chosen offset), were plotted vs time in years back to 1930. The data were averaged over the northern Atlantic latitudes where hurricanes form, over the appropriate time period. (Data from the other tropical basins are of lower availability and quality, so Emanuel's work focused on, but was not limited to, northern hemisphere storms. Those total data cover hurricanes over 70 years in tropical portions of the North Atlantic, North Pacific, West Pacific, and Indian Oceans.) Remarkably detailed fits were shown, with two accurately overlain curves vs time, representing the PDI, a quantity rising from zero to with values in units of $10^{11} \mathrm{~m}^{3} / \mathrm{s}^{2}$, and "temperature". The "temperature" curve was based on accurate ocean temperature measurements, but a carefully chosen adjustable constant was subtracted from the measured temperatures, remarkably to allow the resulting curve to closely overlap the PDI curve. The resulting fits obviously are of the form $\mathrm{PDI}=$ const $(\mathrm{T}-\mathrm{Tc})$, but neither this formula nor the fitting constant Tc were shown in the original papers. This formula is still absent from literature on hurricanes apart from Wolf 2020.

The accurate fits in all basins were accomplished by multiplying PDI by a scale factor, seen as 1/a in Eq. 3 and by subtracting an offset temperature, identified as Tc in Eq. 3. The fitting procedure was first recognized by Wolf 2020 as representing Eq. 3 as a fitting algorithm. This algorithm was very successful applying accurately to storms beyond the North Atlantic basin. For each basin, a single value of Tc was found to apply over periods of decades and hundreds of storms. Emanuel 2005, 2007 did not tabulate values of the Tc nor the scale factors, and in particular did not identify Tc, the temperature at which the PDI "turns off" as a critical temperature for a hurricane. By inspecting the graphs in Emanuel's papers one can see that the Tc value for the North Atlantic is precisely $26.5 \mathrm{C}$ and that the variations of Tc in the other basins, where the data are not so good, allow a small range of values around $26.5 \mathrm{C}$.

From a simple physics point of view the Tc critical temperature for a hurricane, is largely determined by the properties of water, in particular its vapor pressure, but small variations in Tc might well come from systematic changes in the atmosphere characteristics of the separate ocean basins. Wolf 2020 pointed out that the form of Eq 3, in conjunction with the knowledge of a hurricane threshold or transition temperature dating back to the work of Palmen in 1948, were consistent with a hurricane phase transition, hurricanes as a 
separate phase of matter. Such an idea is supported also by the detailed structures, particularly the eye and eyewall regions, following accurate circular forms uncharacteristic of the underlying thunderstorms.

\subsection{Order Parameters for Hurricanes}

It was established by Wolf [2] that the PDI also represents a hurricane order parameter. In the language of a phase change, an order parameter is a quantity that is zero below the transition and measures the strength of the order in the new phase. It is specifically stated by Goldenfeld [5]: "(the) order parameter for a given system is not unique". As examples, the basic order parameter for the ferromagnetic transition is the magnetization $\mathrm{M}$, but $\mathrm{M}^{2}$ and $\mathrm{M}^{3}$ are also possible order parameters.

Coming back to hurricanes, it is plausible that the azimuthal windspeed $\mathrm{v}$ is the basic order parameter and that the measured quantity, PDI, scaling as $\mathrm{v}^{3}$ is also a suitable order parameter, as well as a predictor of potential damage. Thus it appears that the large and accurate data sets for the PDI vs time $[3,4]$ represent the order parameter $\mathrm{v}^{3}$.

\subsection{The Hurricane as a Phase of Matter}

"Critical aspects" of hurricanes are those aspects related to a second-order phase transition. Broadly speaking, a "phase" is a general organization of matter, for example as a solid or a liquid.

The most familiar critical aspects are a transition temperature and critical exponent, occurring in a typical power law relation such as Eq. 3. We (Wolf, 2020) have recently identified a critical temperature for hurricanes. That paper [2] estimated the transition temperature as $26.5 \mathrm{C}$ and the critical exponent related to the windspeed as near $1 / 3$. Thus,

$$
\mathrm{V}=\mathrm{b}\left(\mathrm{T}-\mathrm{T}_{\mathrm{c}}\right)^{\beta}
$$

with $b$ arbitrary and $\beta$ near $1 / 3$.

An excellent review entitled "100 Years of Progress in Tropical Cyclone Research" (2018) by Prof. Kerry Emanuel [6] does not include such a windspeed formula, but describes in detail the "potential intensity", a theoretical estimate of the wind speed possible in the situation of the hurricane. This quantitiy is widely quoted, but is not obviously compatible with the data shown in Figure 1 or with Eq. 4.

"Critical phenomena" have been intensely studied with discovery of subtle mathematical relationships leading to a Nobel Prize in Physics in 1982 for Kenneth G. Wilson [7].

The overlap of this field with meteorology has been limited to the discovery of critical aspects of rainfall by Peters, Neelin and others [8-10], and the Wolf 2020 discovery regarding hurricanes.

The familiar transitions between solid, liquid and gas phases of matter are first order transitions, with discontinuous change in properties such as density at the transition temperature, and requiring a latent heat for the transition to occur. The vaporization of water requires 540 cal/ g. In contrast, the critical phenomena related to rainfall and hurricanes are continuous phase transitions, of the second order, with no latent heat needed.

\section{Second Order Phase Transitions, Ferromagnetism}

Ferromagnetism is perhaps the most familiar example of a continuous phase transition. Iron metal spontaneously becomes magnetic when cooled below $770 \mathrm{C}$, so it will act like a bar magnet. The origin is an internal change in the organization of the electron motions in the iron, and ferromagnetism can be described as a new state where the electron spin moments are aligned. The new state is characterized by its magnetization $\mathrm{M}$, the order parameter, the density of magnetic moments per unit volume. This quantity appears continuously at $T_{c}$ and rises rapidly below $\mathrm{T}_{\mathrm{e}}$. The new state is one of "broken symmetry", because a new direction, that of the locked-together spins, has spontaneously appeared. The further characteristic is that the magnetization, the order parameter of the new state, rises as a power law $\left(T_{c}-T\right)^{\beta}$, where $\beta$ is the critical exponent. An added fact is that the basic magnetic moment $\mu=\mathrm{iA}$, defined as an area $\mathrm{A}$ times a loop current $\mathrm{I}=\mathrm{dq} / \mathrm{dt}$, is related to an angular momentum $\mathrm{L}$ by the gyromagnetic ratio e/2m. Thus we have $\mu=(\mathrm{e} / 2 \mathrm{~m}) \mathrm{L}$, where e and $\mathrm{m}$, respectively are particle charge and mass.

An important special case, uniaxial antiferromagnetism, turns out to be closely related to the hurricane transition, through its connection with an ideal uniaxial antiferromagnet (IUA) also known as the "Ising model". An antiferromagnet is a system with two opposite but unequal moments in each unit cell. A uniaxial system is one where the magnetization is directed only along a particular direction, say the $\mathrm{z}$ direction. The IUA is a linear array of equal moments, with nearest neighbor interaction, that each can be directed in either the plus or minus $\mathrm{z}$ direction. It has been well established, see for example the book [5] of Goldenfeld, that the uniaxial antiferromagnet $\mathrm{DyAlO}_{3}$ with critical exponent measured as 0.311 is an experimental realization of the IUA. The important possibility here is of a universal critical exponent number that is distinct from $1 / 3$, that will apply to hurricanes. Following Goldenfeld, one might take that exponent, 0.311, as the experimental value for the IUA. However, for the IUA (Ising Model) an authoritative review (Polisetto and Vicari 2002) [11] has given a theoretical value of this exponent as 0.3265. In their paper are also quoted 21 separate experimental values of the exponent $\beta$ rangeing from 0.111 to 0.341 , and whose average value is 0.3197 . Taking all these more recent values into account, we suggest the Isiing model universality class critical exponent as $0.323 \pm 0.01$, and suggest that this number is In agreement, within the various errors involved, with our nominal observed value $1 / 3$ for the hurricane phase. The experts Polisetto and Vicari state [11] that the renormalization group approach of K. G. Wilson "explains......why fluids and uniaxial antiferromagnets behave in an identical way at the critical point", certainly to imply the exponents are identical. So there is a possibility 
that the value 0.323 , that we attribute to the universality class, is the preferred value. In that case the critical exponent for the wind speed is $0.323 \pm 0.01$ and that for the PDI is $0.969 \pm 0.03$. These values are consistent with the inexact available data for PDI vs temperature shown in Figure 1 and also the extended data shown in Emanuel 2005, 2007.

\section{Hurricane Universality Class}

It appears that the magnetization in the IUA is a prototype for the windspeed of a hurricane. This Ising model behavior is illustrated in Figure 2, (Holmes et al 1971) by the antiferromagnet $\mathrm{DyAlO}_{3}$, where the measured quantity, proportional to the magnetization order parameter, is the magnetoelectric effect $\alpha$. In Figure 2 the ordinate is the magnetoelectric constant at temperature $\mathrm{T}$, normalized by its value at $1.4 \mathrm{~K}$, well below the transition temperature, called the Neel temperature $T_{N}=3.525$. As explained in detail by Goldenfeld (1992), this antiferromagnetic system is reliable as representing typical behavior near the transition of the IUA. Further, the exponent found, 0.311, should not only apply to all uniaxial anti- ferromagnets but also to a set of other systems unrelated to magnetism but in the same "universality class". Such a separate system known to be in the Ising class is the liquid-vapor transition in simple fluids $[5,7,8]$. The value of the critical exponent in each universality class can be arrived at using the "renormalization group". This generality is surprising, and led to the Nobel Prize. The consequences of this relatively new body of knowledge for rainfall and hurricanes are only beginning to be explored.

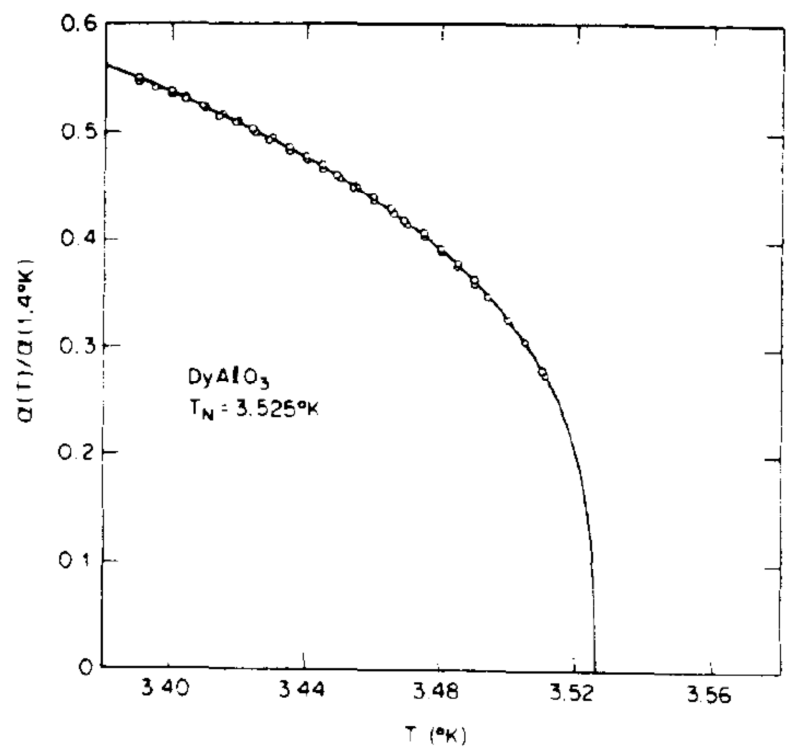

Figure 2. Power law fit to magnetic transition for uniaxial anti-ferromagnet $\mathrm{DyAlO}_{3}$ showing with precision that the critical exponent is 0.311 , suggested as applicable to the Ising model (IUA) universality class. Holmes et al 1971.

The main result is that the order parameter for the exemplary antiferromagnet $\mathrm{Dy} \mathrm{AlO}_{3}$ is shown by Holmes et al [12] to vary as const $\left(\mathrm{T}_{\mathrm{N}}-\mathrm{T}\right)^{0,311}$, to high accuracy, near the transition point. The high accuracy of the fit is seen in Figure 2, extending to $3.39 \mathrm{~K}$ from $3.525 \mathrm{~K}$. This range, $0.135 \mathrm{~K}$, is 0.038 of $\mathrm{T}_{\mathrm{N}}$. The equivalent temperature range, where the power law is similarly expected to be extremely accurate, for the hurricane case, with $\mathrm{T}_{\mathrm{c}}=26.5 \mathrm{C}=299.5 \mathrm{~K}$, would then be $11.4 \mathrm{~K}$, extending the expected range of accuracy of the power law fit (see Figure 1) to $37.9 \mathrm{C}$ or $112.3 \mathrm{~F}$. Universality has been invoked for the following reasons. First, Wolf 2020 showed that the exponent for the PDI is nominally unity. Since the PDI scales as the cube of the velocity, the more fundamental exponent, that for the velocity, is nominally 0.333 , close to that for the Ising model and Figure 2. Second, one can argue that the hurricane is in the same universality class as the magnetic Ising model because one can essentially map the Hamiltonian of the hurricane onto that of the Ising model by multiplying by the gyromagnetic ratio e $/ 2 \mathrm{~m}$. The magnet is a vertically directed moment and the hurricane, via the gyromagnetic ratio is a vertically directed angular momentum vector $\mathrm{L}=\mathrm{Mvr}$. If the two cases are in the same universality class we can literally think of rewriting the ordinate scale of Figure $2 \mathrm{in} \mathrm{m} / \mathrm{s}$ and changing the temperature scale to run from $40 \mathrm{C}$ to the transition at $26.5 \mathrm{C}$. The basic physics allows one to state that the precision seen in the antiferromagnetic fit will be expected for the hurricane case, that is of course impossible to accurately measure since one cannot create hurricanes in the laboratory, while watching the thermometer. If this is correct, one can be confident that the power-law-dependence is adequate for any reasonable scenario of global warming, below sea temperatures of $112 \mathrm{~F}$. This view relegates the extensive potential intensity theory to possibly describing a high temperature asymptotic region that, however, is unlikely to be reached. All of this agrees with the well-known fact (Dare and McBride, 2011) [13] that hurricanes do not form below $26.5 \mathrm{C}$ ocean temperature.

\section{Precise Prediction of Hurricane Power vs Ocean Temperature}

Following the discussion above, a precise prediction of the PDI vs ocean temperature is

$$
\mathrm{PDI}=\mathrm{a}\left(\mathrm{T}-\mathrm{T}_{\mathrm{c}}\right)^{0.969 \pm 0.03}
$$

where $T_{c}=26.5 \pm 0.01 \mathrm{C}$ and a is arbitrary. The precise value is based on the critical exponent for the Ising Model, but, with the error range, overlaps the simple statement of linearity following from Figure 1 and also overlaps the value that follows from the L. M. Holmes data of Figure 2.

Correspondingly, the precise value for the maximum windspeed is

$$
\mathrm{V}=\mathrm{b}\left(\mathrm{T}-\mathrm{T}_{\mathrm{c}}\right)^{0,323 \pm 0.01}
$$

with $\mathrm{b}$ arbitrary. The analysis given in conjunction with Figure 2 suggests that these formulas should be accurate for sea surface temperatures as high as $38 \mathrm{C}$, a temperature range that includes any likely climatic future. 


\section{Predicting Future Hurricane Power}

The replotted PDI data $[2,4]$ here shown in Figure 1, confirm Eqs. 3 and 5, in the straight line shown extrapolating to the critical temperature of $26.5 \mathrm{C}$, a value in close agreement with Dare and McBride. This line in Figure 1 increases from PDI near 1.5 (PDI in units $10^{11} \mathrm{~m}^{3} \mathrm{~s}^{-2}$ ) at $26.85 \mathrm{C}$ to about 4 at $27.6 \mathrm{C}$. That is a rate of change in PDI of $3.33 \times 10^{11} \mathrm{~m}^{3} \mathrm{~s}^{-2}$ per degree. If we extrapolate 2 degrees higher, say to year 2100, from the value 4 near 2005 we find $\mathrm{PDI}=10.66$, that is a factor $10.66 / 4=2.67$ larger. So the PDI roughly triples if there is a $2 \mathrm{C}$ increase in sea temperature. In terms of windspeed the increase will be proportional to the cube root, thus an increase in windspeed by a factor $(2.67)^{1 / 3}=1.386$. So the maximum wind scale would be increased by $39 \%$ while the expected monetary losses, scaling with power expended would nearly triple, increasing by a factor 2.67 .

\section{Conclusions}

In conclusion, we confirm an alternative view [2] of extensive and closely fitted data sets [3, 4] of the hurricane Power Dissipation Index vs measured sea surface temperature T. These data provide excellent detailed fits if the measured temperatures are subjected to offsets that are equivalent to choosing a critical temperature for a tropical cyclone, via fitting law Eq. (3), with linear dependence on $\left(T-T_{c}\right)$. The inferred Eq. 3 is confirmed by direct data replotted in Figure 1. It is suggested that the data and fits are strong evidence that appearance of hurricanes can usefully be viewed as a critical point, of the macroscopic warm ocean-atmosphere system. This also reveals a stronger temperature dependence of the cyclone strength, represented by the PDI, that seems of interest and concern from the point of view of rising ocean temperature. We here find that precise values of the critical exponents for the PDI and maximum windspeed, respectively, are $0.969 \pm 0.03$ and $0.323 \pm 0.01$. These slight improvements on our earlier values [2] are found by recognizing the hurricane phase transition to be in the Ising Model universality class, known for the transition of the uniaxial antiferromagnet (Figure 2) and the liquid-vapor transition of simple fluids. It is hoped that recognition of the hurricane as a phase of matter with the universality class of the Ising Model will help in resolving remaining questions on the formation of these storms, such as noted by Smith and Montgomery [14, 15].

\section{Funding}

This research received no external funding.

\section{Conflict of Interest}

The author declares no conflict of interest.

\section{Acknowledgements}

The author thanks S. Arnold, K. Becker, K. R. Sreenivasan and O. Pauluis for helpful discussions. He also thanks Lewis M. Holmes for permission to reproduce a figure from his paper of 1971 that appears as Figure 2 above. He thanks Professor Kerry Emanuel for helpful correspondence and useful comments during the review of this paper. This work was supported by the NYU Tandon School of Engineering through its Department of Applied Physics. The author particularly acknowledges the support of Prof. John Di Bartolo.

\section{References}

[1] Palmén, E., 1948: On the formation and structure of tropical hurricanes. Geophysica, 3, 26-39.

[2] Wolf, E. (2020) "Critical Behavior of Tropical Cyclones", Theoretical and Applied Climatology, 139 (3) 1231.

[3] K. Emanuel, Increasing destructiveness of tropical cyclones over the past 30 years. Nature 436, 68 (2005).

[4] Kerry Emanuel, Environmental factors affecting tropical cyclone power dissipation. J. Climate 20, 5497 (2007).

[5] Nigel Goldenfeld, Lectures on Phase Transitions and the Renormalization Group. Taylor and Francis (1992). See Section 5.3 .

[6] Emanuel, Kerry (2018) "100 Years of Progress in Tropical Cyclone Research", Ch. 15 in AMS Monographs, Vol. 59 (American Meteorological Society).

[7] Wilson, K. (1983) "The renormalization group and critical phenomena" Reviews of Modern Physics 55, 583.

[8] Peters, O. M. and J. D. Neelin. (2006) Critical phenomena in atmospheric precipitation. Nature Physics 2, 393.

[9] Neelin, J., Peters, O., Lin, J., Hales, K., and Holloway, C. (2008) "Rethinking Convective Quasi-equilibrium: observational constraints for stochastic convective schemes in climate models” Phil. Trans. Royal Soc. A 366, 2581.

[10] Peters, O. and Neelin, J. (2009) "Atmospheric Convection as a Continuous Phase Transition: Further Evidence" Int. J. Mod. Phys. B 23, 5453.

[11] Polisetto, A. and Vicari, E. (2002) "Critical Phenomena and Renormalization Group Theory" Physics Reports 368, 547.

[12] L. M. Holmes, L. G. Van Uitert, and G. W. Hull (1971). "Magnetoelectric effect and critical behavior in the Ising-like antiferromagnet $\mathrm{DyAlO}_{3}$ " Solid State Communications 9, 1373.

[13] R. Dare and J. L. McBride. The threshold sea surface temperature condition for tropical cyclogenesis. J. of Climate 24, 4570-4576 (2011).

[14] Smith, R., Montgomery, M., and Van Sang, N. (2009) "Tropical cyclone spin-up revisited" Q. J. R. Meteorol. Soc. $135,1321$.

[15] Smith, R and Montgomery, M. (2016). "Understanding Hurricanes" Weather 71, 219. 